05

\title{
Воздействие поля зонда магнитно-силового микроскопа на скирмионное состояние в модифицированной пленке Co/Pt c перпендикулярной анизотропией
}

\author{
(С) В.Л. Миронов ${ }^{1}$, Р.В. Горев ${ }^{1}$, О.Л. Ермолаева ${ }^{1}$, Н.С. Гусев ${ }^{1}$, Ю.В. Петров ${ }^{2}$ \\ ${ }^{1}$ Институт фризики микроструктур РАН, \\ Нижний Новгород, Россия \\ ${ }^{2}$ Санкт-Петербургский государственный университет, \\ Санкт-Петербург, Россия \\ E-mail: mironov@ipmras.ru
}

Поступила в Редакцию 15 апреля 2019 г.

В окончательной редакции 22 апреля 2019 г.

Принята к публикации 24 апреля 2019 г.

\begin{abstract}
Приводятся результаты экспериментов по локальному перемагничиванию зондом магнитно-силового микроскопа искусственных магнитных скирмионов в многослойной пленке $\mathrm{Co} / \mathrm{Pt}$ с перпендикулярной намагниченностью. Образец представлял собой пленку $\mathrm{Co} / \mathrm{Pt}$, содержащую массив областей цилиндрической формы с пониженной анизотропией, модифицированных фокусированным ионным пучком $\mathrm{He}^{+}$. Магнитное состояние образца контролировалось методами магнитно-силовой микроскопии. Перемагничивание осуществлялось полем зонда МСМ при проходах над образцом на малых высотах. Методами микромагнитного моделирования исследованы эффекты взаимодействия поля МСМ зонда со скирмионным состоянием намагниченности в данных структурах.
\end{abstract}

Ключевые слова: магнитно-силовая микроскопия, магнитный скирмион, эффекты перемагничивания, микромагнитное моделирование.

DOI: $10.21883 /$ FTT.2019.09.48104.07N

\section{1. Введение}

Многослойные тонкопленочные структуры с перпендикулярной анизотропией привлекают большое внимание в связи с перспективностью их использования в качестве рабочих слоев в приборных структурах спинтроники, а также в магнитных системах хранения и обработки информации [1]. Обычно наноструктурирование пленочных образцов производится посредством изменения их топологии методами литографии. Однако в последние годы были развиты новые методы наноструктурирования, основанные на облучении образцов с помощью фокусированных ионных пучков [2,3], что позволяет локально модифицировать свойства пленочных структур без изменения их рельефа. Особенно эффективен данный подход в применении к многослойным тонкопленочным структурам $\mathrm{Co} / \mathrm{Pt}$ с перпендикулярной намагниченностью, в которых с помощью фокусированных ионных пучков удается локально модифицировать параметр перпендикулярной анизотропии [4-7]. В частности, в работе [8] было показано, что данный метод позволяет создавать плотные решетки искусственных магнитных скирмионов, которые могут быть применены в качестве среды для записи информации с высокой плотностью.

Одним из методов селективного управления магнитным состоянием ферромагнитных наноструктур является их локальное перемагничивание с помощью неоднородного поля зонда магнитно-силового микроскопа
(MCM) [9]. Выбирая зонды с достаточно большим магнитным моментом, в эксперименте удается реализовать локальное переключение намагниченности наноструктур при сканировании на малых высотах и впоследствии контролировать результаты перемагничивания, регистрируя МСМ изображения на относительно больших расстояниях зонд-образец. В частности, данная методика была использована нами при исследовании особенностей МСМ перемагничивания наноостровков $\mathrm{Co} / \mathrm{Pt}[10]$. В настоящей работе эта методика применена для селективного перемагничивания искусственных скирмионов в модифицированной пленке $\mathrm{Co} / \mathrm{Pt}$.

\section{2. Эксперимент}

Исследовались многослойные структуры $[\mathrm{Co}(0.5 \mathrm{~nm}) / \operatorname{Pt}(1 \mathrm{~nm})]_{5}$, выращенные на $\mathrm{Si}(100)$ подложках с буферным подслоем $\mathrm{Ta}(10 \mathrm{~nm}) / \mathrm{Pt}(10 \mathrm{~nm})$ методом магнетронного осаждения. Данные структуры были пространственно модифицированы фокусированным ионным пучком $\mathrm{He}^{+}$в микроскопе „Orion“ с приставкой „Nanomaker“ (Carl Zeiss, Jena). Облучение образцов производилось растровым методом. Диаметр фокусированного пучка ионов составлял $5 \mathrm{~nm}$, энергия ионов - $30 \mathrm{keV}$, доза облучения $10^{15}$ ions $/ \mathrm{cm}^{2}$. В результате в пленке был сформирован массив $20 \times 14 \mu \mathrm{m}^{2}$, состоящий из модифицированных областей цилиндрической формы диаметром $100 \mathrm{~nm}$, 

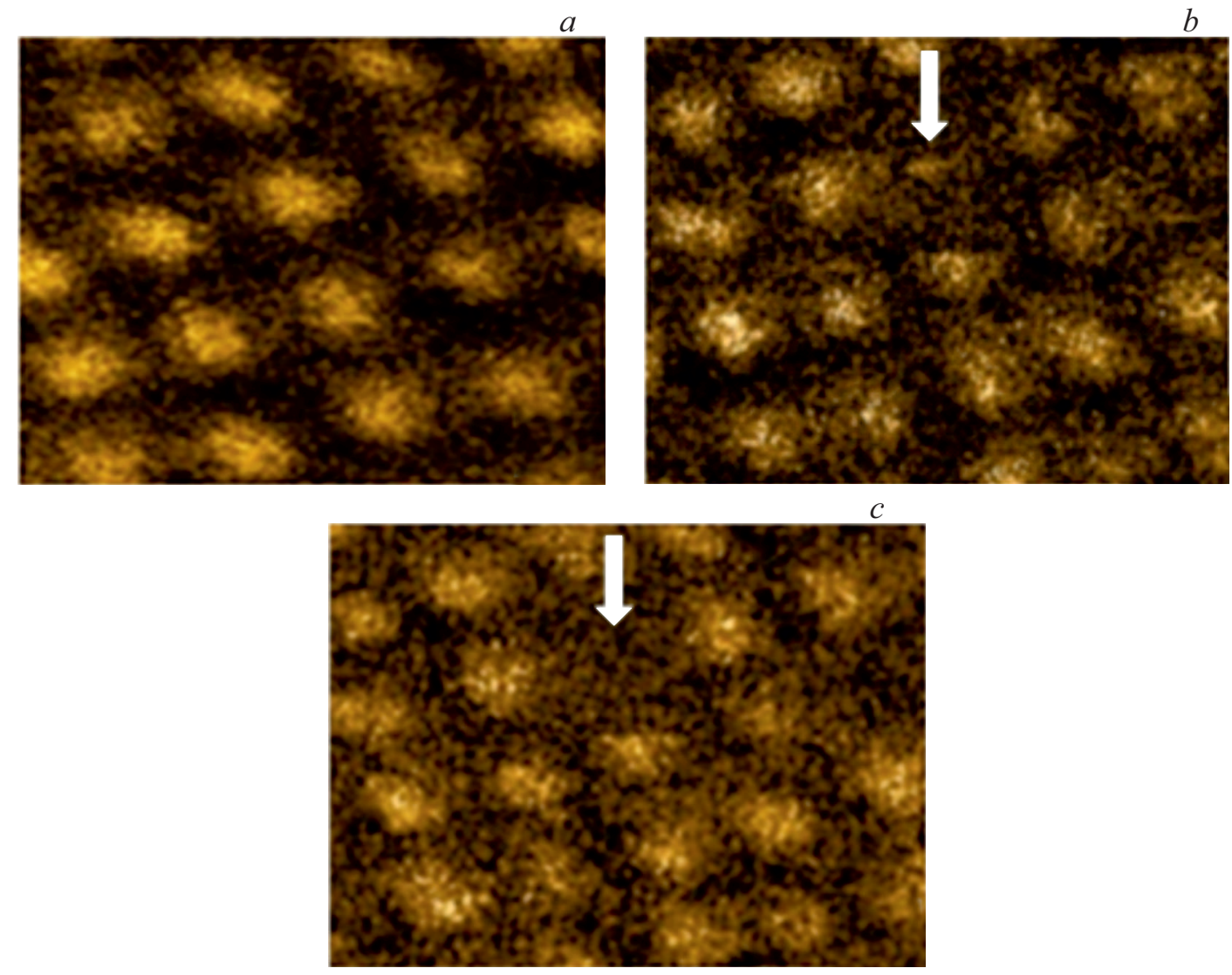

Рис. 1. Последовательные МСМ изображения одного и того же участка массива скирмионов в Со/Рt. $a-$ Начальное состояние. MCM изображение при проходе на высоте $30 \mathrm{~nm} . b-\mathrm{MCM}$ изображение при проходе на высоте $15 \mathrm{~nm} . c-$ Конечное состояние. Стрелками показана позиция скирмиона, который изменил направление намагниченности во время сканирования на малой высоте. Размер кадров $900 \times 700 \mathrm{~nm}$.

имеющих пониженную анизотропию и упорядоченных на квадратной решетке с периодом $200 \mathrm{~nm}$ [8].

Магнитное состояние образца и эффекты перемагничивания исследовались с помощью зондового магнитносилового микроскопа „Solver-HV“ (НТ-МДТ, Зеленоград). В качестве зондового датчика использовался стандартный кантилевер NSG-03, покрытый слоем Со толщиной $30 \mathrm{~nm}$. Перед измерениями зонд намагничивался вдоль оси пирамиды во внешнем поле 2 Т. В качестве МСМ отклика регистрировался сдвиг фазы вынужденных колебаний кантилевера (возбуждаемых на резонансной частоте), вызванный градиентом поля рассеяния образца, при проходе на постоянной высоте.

В эксперименте вначале образец намагничивался до насыщения во внешнем перпендикулярном магнитном поле 500 Ое. Затем прикладывалось инвертированное поле $200 \mathrm{Oe}$, и в модифицированной пленке $\mathrm{Co} / \mathrm{Pt}$ формировалась решетка скирмионов (рис. 1,a), так что намагниченность в скирмионах и магнитный момент зонда были направлены в противоположных направлениях. Невозмущенные МСМ изображения образца получались при проходе на больших высотах (расстояние между кончиком зонда и образцом > $30 \mathrm{~nm}$, амплиту- да колебаний зонда $\sim 10 \mathrm{~nm})$. При перемагничивании среднее расстояние зонд-образец сокращалось вручную в процессе сканирования до $15 \mathrm{~nm}$, и наблюдались единичные переключения намагниченности скирмионов, сопровождающиеся резким изменением МСМ контраста (рис. $1, b$ и $c$ ).

\section{3. Микромагнитное моделирование}

Нами было проведено микромагнитное моделирование процесса перемагничивания скирмиона в поле зонда МСМ. Моделирование проводилось с помощью пакета Object Oriented MicroMagnetic Framework (OOMMF) [11] для квадратного участка пленки $\mathrm{Co} / \mathrm{Pt}$ размером $200 \times 200 \mathrm{~nm}$, содержащего область с пониженной анизотропией диаметром $100 \mathrm{~nm}$. Толщина пленки $\mathrm{Co} / \mathrm{Pt}-7.5 \mathrm{~nm}$. В расчетах использовались усредненные материальные параметры пленок $\mathrm{Co} / \mathrm{Pt}[8,12,13]$ : намагниченность насыщения - $4 \cdot 10^{5} \mathrm{~A} / \mathrm{m}$, обменная константа $-2 \cdot 10^{12} \mathrm{~J} / \mathrm{m}$. Поле анизотропии плёнки $\mathrm{Co} / \mathrm{Pt}$ выбиралось равным $413 \mathrm{kA} / \mathrm{m}$, в модифицированной области его величина была уменьшена до $404 \mathrm{kA} / \mathrm{m}$. Размер ячейки разбиения $2.5 \times 2.5 \times 7.5 \mathrm{~nm}$. Вначале 


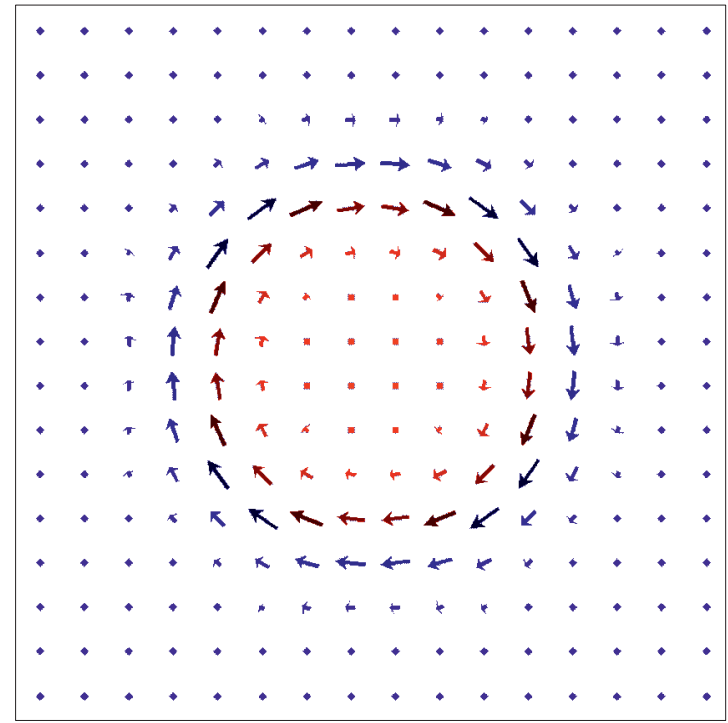

Рис. 2. Распределение намагниченности на участке $200 \times 200 \mathrm{~nm}$ пленки $\mathrm{Co} / \mathrm{Pt}$, соответствующее скирмионному состоянию в модифицированной области диаметром $100 \mathrm{~nm}$.

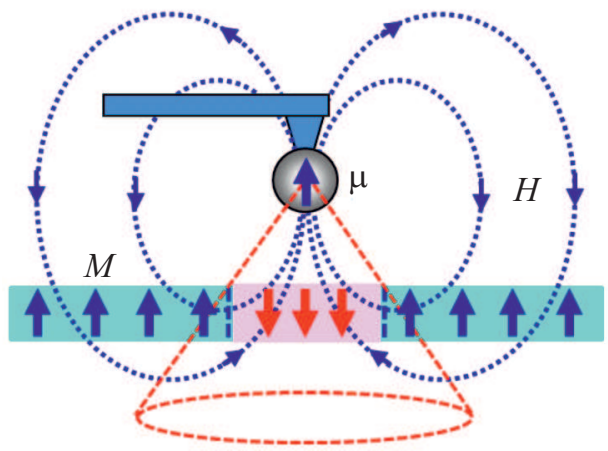

Рис. 3. Схематическое изображение системы зонд-образец.

образец намагничивался до насыщения во внешнем перпендикулярном магнитном поле 500 Ое. Затем прикладывалось инвертированное поле $200 \mathrm{Oе}$, и в модифицированной области пленки $\mathrm{Co} / \mathrm{Pt}$ формировался искусственный скирмион, представляющий собой домен с инвертированной намагниченностью (рис. 2). Граница между областями с противоположно направленной намагниченностью представляет собой блоховскую доменную стенку.

В модельных расчетах поле зонда, представлялось полем точечного магнитного диполя с магнитным моментом $\mu$, равным магнитному моменту однородно намагниченного шара из Со диаметром $50 \mathrm{~nm}$ [10]. Магнитное поле такого зонда имеет аксиально-симметричную структуру (рис. 3). При этом z-составляющая поля меняет свой знак на поверхности конуса, показанного на рисунке пунктиром. Эффекты перемагничивания скирмиона определяются величиной магнитного момента зонда и его положением над модифицированной областью.
Перемагничивание скирмиона возможно двумя способами. В первом случае перемагничивание производится при вертикальном опускании зонда в центр модифицированной области. При этом вначале зонд вдали от образца, и при сближении на определенное расстояние (критическое расстояние для перемагничивания) происходит изменение направления намагниченности в модифицированной области. На рис. 4 приведены последовательные стадии перемагничивания скирмиона. Из рисунка видно, что при сближении зонда с образцом происходит постепенное уменьшение диаметра домена с инвертированной намагниченностью (рис. 4,a) и превращение его в магнитный вихрь (рис. 4, $b$ и $c$ ), а затем наблюдается полное перемагничивание (рис. $4, d$ ). Затравкой для перемагничивания служит блоховская доменная стенка на границе домена с инвертированной намагниченностью (рис. 2). Данный процесс качественно отличается от сценария перемагничивания нанодисков $\mathrm{Co} / \mathrm{Pt}$ в поле зонда MCM [10], в котором магнитный вихрь зарождается в центре диска и волна перемагничивания распространяется от центра к периферии.

Второй способ перемагничивания реализуется, при продольном движении МСМ зонда над скирмионом. В этом случае критическая высота, при прохождении на которой происходит перемагничивание, имеет меньшее значение, поскольку взаимодействие зонда и скирмиона носит более сложный характер. В качестве примера, на рис. 5 и 6 приведены последовательные стадии изменения распределения намагниченности при продольном
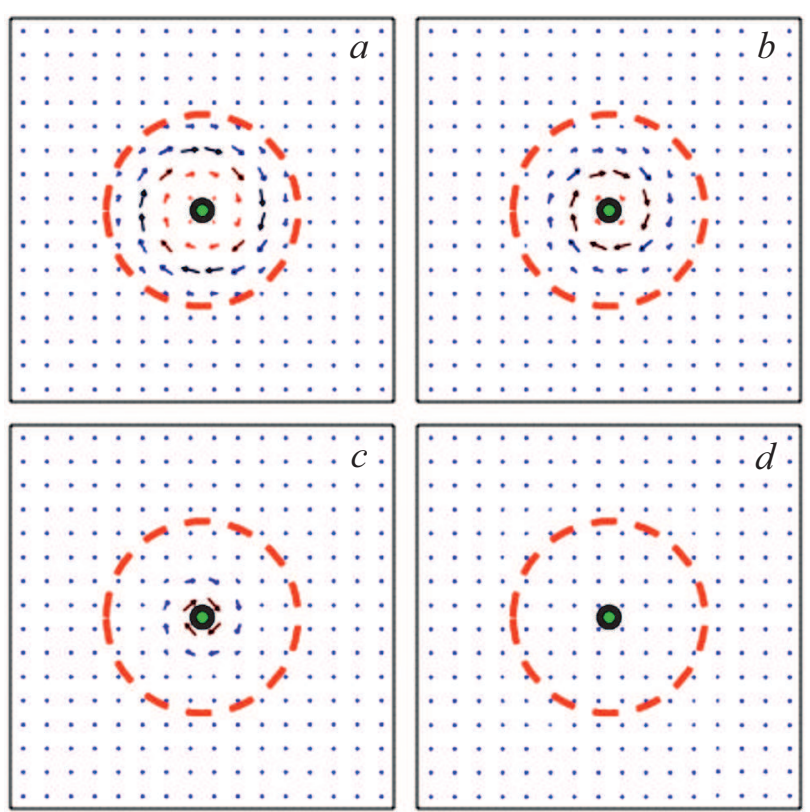

Рис. 4. Последовательные стадии перемагничивания скирмиона при вертикальном сближении зонда с образцом. $a-$ Расстояние от центра зонда до поверхности образца $h=100 \mathrm{~nm}$, $b-h=90 \mathrm{~nm}, c-h=80 \mathrm{~nm}, d-h=60 \mathrm{~nm}$. Зонд (обозначен темным кружком) расположен над центром модифицированной области (ее граница показана пунктирной линией). 

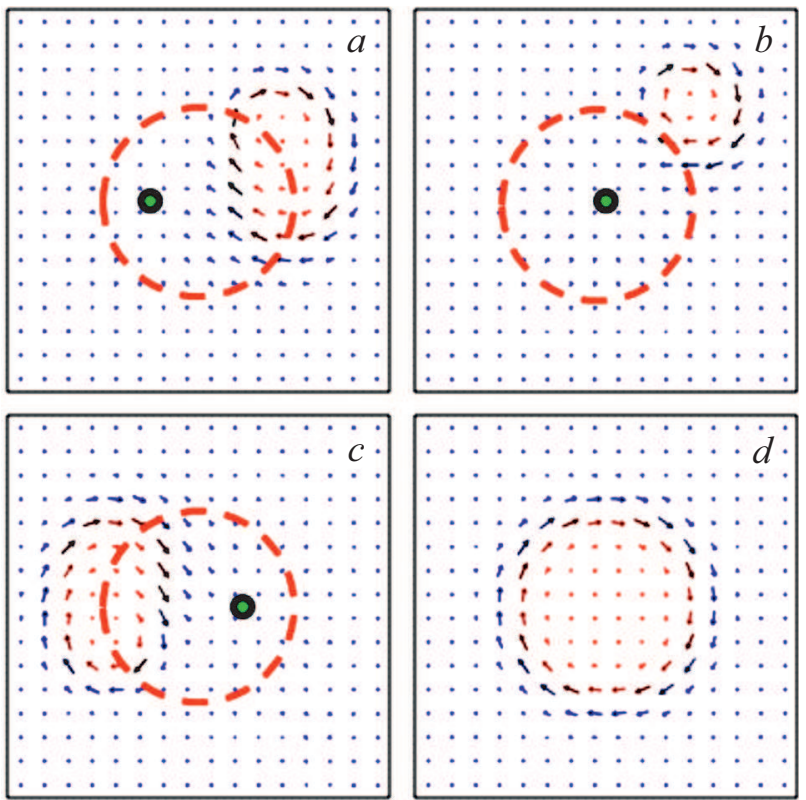

Pис. 5. Последовательные стадии прохождения МСМ зонда над центром модифицированной области пленки $\mathrm{Co} / \mathrm{Pt}$ со скирмионным состоянием. Высота прохода (расстояние от центра зонда до поверхности образца) равно $55 \mathrm{~nm}$
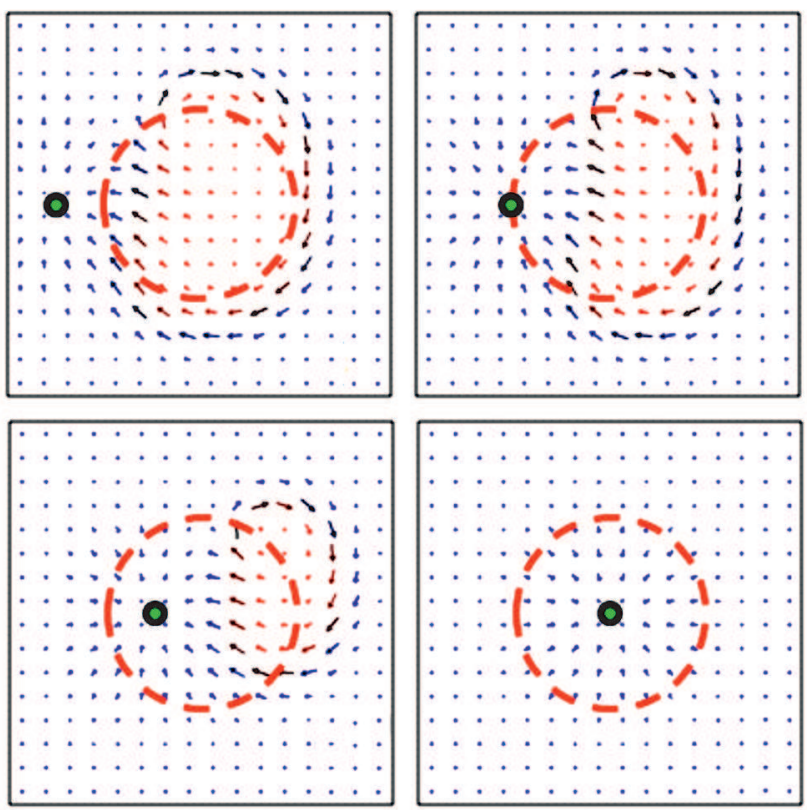

Рис. 6. Последовательные стадии прохождения МСМ зонда над центром модифицированной области со скирмионным состоянием в пленке $\mathrm{Co} / \mathrm{Pt}$. Высота прохода (расстояние от центра зонда до поверхности образца) равно $40 \mathrm{~nm}$.

прохождения МСМ зонда над модифицированной областью на различных высотах. При прохождении на достаточно большой высоте (больше критической) перемещение зонда с периферии моделируемого участка пленки к центру приводит к смещению инвертированного домена на границу модифицированной области (рис. 5, $a$ и $b$ ), где происходит его частичное пиннингование. Существование скирмиона в этом случае поддерживается $z$-компонентой поля зонда вне конуса (см. рис. 3). При дальнейшем движении зонда скирмион смещается по направлению против часовой стрелки вдоль границы модифицированной области и затем возвращается в центр (рис. 5,d). Аналогичные процессы наблюдались при моделировании взаимодействия МСМ зонда с магнитным вихрем в дисках из материалов с плоскостной анизотропией, где эффекты смещения вихря также были обусловлены взаимодействием поля зонда с кором и вихревой оболочкой [14].

Другая ситуация наблюдается при прохождении зонда на высоте, меньше критической. На рис. 6 показаны последовательные стадии прохождения зонда над образцом на высоте $40 \mathrm{~nm}$. Вначале скирмион испытывает сильное возмущение и вытесняется полем зонда на границу модифицированной области (рис. 6, $a-c$ ), a затем происходит перемагничивание пленки с переходом модифицированной области из скирмионного состояния в состояние с однородной намагниченностью (рис. $6, d)$, которое и сохраняется при удалении зонда. По-видимому, аналогичный процесс имеет место при перемагничивании скирмиона в эксперименте при сканировании на малой высоте.

\section{4. Заключение}

Таким образом, проведены МСМ исследования скирмионных состояний в пространственно модифицированных пленках $\mathrm{Co} / \mathrm{Pt}$ с перпендикулярной анизотропией. Показано, что при сканировании образца на малых высотах наблюдаются эффекты перемагничивания скирмиона под действием поля зонда МСМ. Методами микромагнитного моделирования изучены процессы перестройки скирмионного распределения намагниченности, локализованного в области с пониженной анизотропией, в зависимости от положения зонда относительно образца. Результаты моделирования качественно объясняют наблюдаемое в эксперименте перемагничивание скирмионов в процессе сканирования образца на малых высотах.

\section{Финансирование работы}

Работа выполнена при поддержке РФФИ (изготовление модифицированных образцов $\mathrm{Co} / \mathrm{Pt}$ проект 18-02-00827, эксперименты по МСМ перемагничиванию и микромагнитное моделирование - проект 18-02-00247). Использовалось оборудование ЦКП „Физика и технология микро- и наноструктур“ ИФМ РАН и Междисциплинарного ресурсного центра по направлению „Нанотехнологии“ СПбГУ.

\section{Конфликт интересов}

Авторы заявляют, что у них нет конфликта интересов. 


\section{Список литературы}

[1] R.L. Stamps, S. Breitkreutz, J. Akerman, A.V. Chumak, Y. Otani, G.E.W. Bauer, J.-U. Thiele, M. Bowen, S.A. Majetich, M. Klaui, I.L. Prejbeanu, B. Dieny, N.M. Dempsey, B. Hillebrands. J. Phys. D 47, 333001 (2014).

[2] C. Chappert, H. Bernas, J. Ferre, V. Kottler, J.-P. Jamet, Y. Chen, E. Cambril, T. Devolder, F. Rousseaux, V. Mathet, H. Launois. Science 280, 1919 (1998).

[3] T. Devolder, C. Chappert, Y. Chen, E. Cambril, H. Bernas, J.P. Jamet, J. Ferre. Appl. Phys. Lett. 74, 3383 (1999).

[4] T. Devolder, J. Ferre, C. Chappert, H. Bernas, J.-P. Jamet, V. Mathet. Phys. Rev. B 64, 064415 (2001).

[5] C. Vieu, J. Gierak, H. Launois, T. Aign, P. Meyer, J.P. Jamet, J. Ferre, C. Chappert, T. Devolder, V. Mathet, H. Bernas. J. Appl. Phys. 91, 3103 (2002).

[6] A. Aziz, S.J. Bending, H. Roberts, S. Crampin, P.J. Heard, C.H. Marrows. J. Appl. Phys. 98, 124102 (2006).

[7] O.L. Ermolaeva, N.S. Gusev, E.V. Skorohodov, Yu.V. Petrov, M.V. Sapozhnikov, V.L. Mironov. Materials 10, 1034 (2017).

[8] M.V. Sapozhnikov, S.N. Vdovichev, O.L. Ermolaeva, N.S. Gusev, A.A. Fraerman, S.A. Gusev, Yu.V. Petrov. Appl. Phys. Lett. 109, 042406 (2016).

[9] В.Л. Миронов, А.А. Фраерман, Б.А. Грибков, О.Л. Ермолаева, А.Ю. Климов, С.А. Гусев, И.М. Нефедов, И.А. Шерешевский. Физика металлов и металловедение 110, 708 (2010).

[10] V.L. Mironov, B.A. Gribkov, S.N. Vdovichev, S.A. Gusev, A.A. Fraerman, O.L. Ermolaeva, A.B. Shubin, A.M. Alexeev, P.A. Zhdan, C. Binns. J. Appl. Phys. 106, 053911 (2009).

[11] M.J. Donahue, D.G. Porter. Interagency Report No. NISTIR 6376, NIST, Gaithersburg.

[12] M.V. Sapozhnikov, R.V. Gorev, E.A. Karashtin, V.L. Mironov. J. Magnet. Magn. Mater. 446, 1 (2018).

[13] M.V. Sapozhnikov, O.L. Ermolaeva. Phys. Rev. B 91, 024418 (2015).

[14] В.Л. Миронов, О.Л. Ермолаева. Поверхность. Рентгеновские, синхротронные и нейтронные исследования 8, 37 (2007).

Редактор Д.В. Жуманов 